Bond University

Research Repository

\title{
Australian Tactical Loads and their Operational Impacts
}

\author{
Orr, Rob Marc; Pope, Rodney
}

Licence:

CC BY-NC-ND

Link to output in Bond University research repository.

Recommended citation(APA):

Orr, R. M., \& Pope, R. (2017). Australian Tactical Loads and their Operational Impacts. 4th International Congress on Soldiers' Physical Performance, Melbourne, Victoria, Australia.

\section{General rights}

Copyright and moral rights for the publications made accessible in the public portal are retained by the authors and/or other copyright owners and it is a condition of accessing publications that users recognise and abide by the legal requirements associated with these rights.

For more information, or if you believe that this document breaches copyright, please contact the Bond University research repository coordinator. 


\section{Australian Tactical Loads and their Operational Impacts}

\section{Dr Rob Orr (PhD, PHTY, BFET, TSAC-F, ADFPTS)}

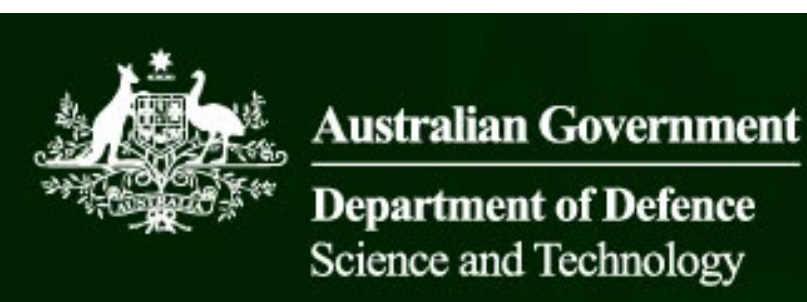

$4^{\text {th }}$ International Congress on Soldiers' Physical Performance 28 November - 1 December 2017

Melbourne Australia

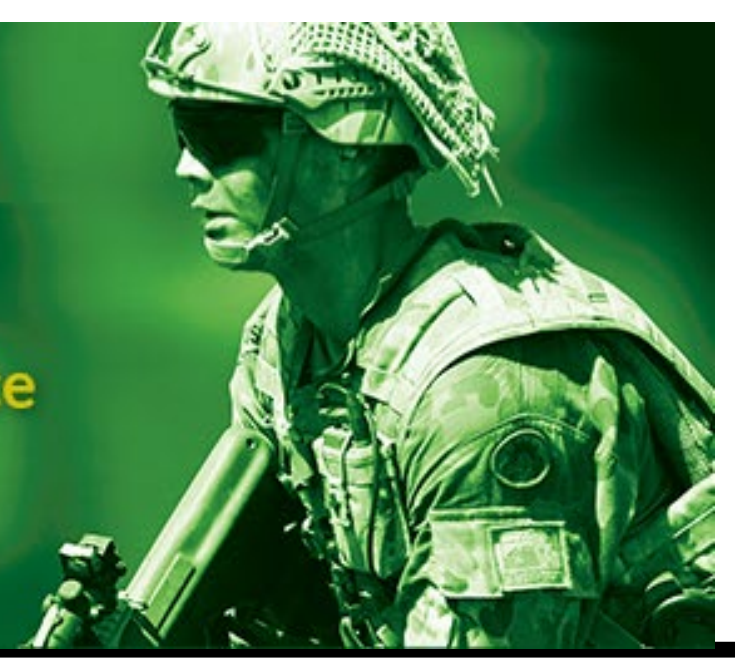




\section{HISTORICAL CONTEXT - MILITARY}

\section{Background}

- From the early Assyrian spearman of antiquity (circa 800 B.C.), soldiers have been required to carry external loads consisting of weaponry, equipment and food

(Orr, 2010; Knapick et al., 2012:2004)

- Downstream effects of these loads have been shown to impact on the tactics of warfare, cause injury and reduce fighting force size 


\section{HISTORICAL CONTEXT - MILITARY}

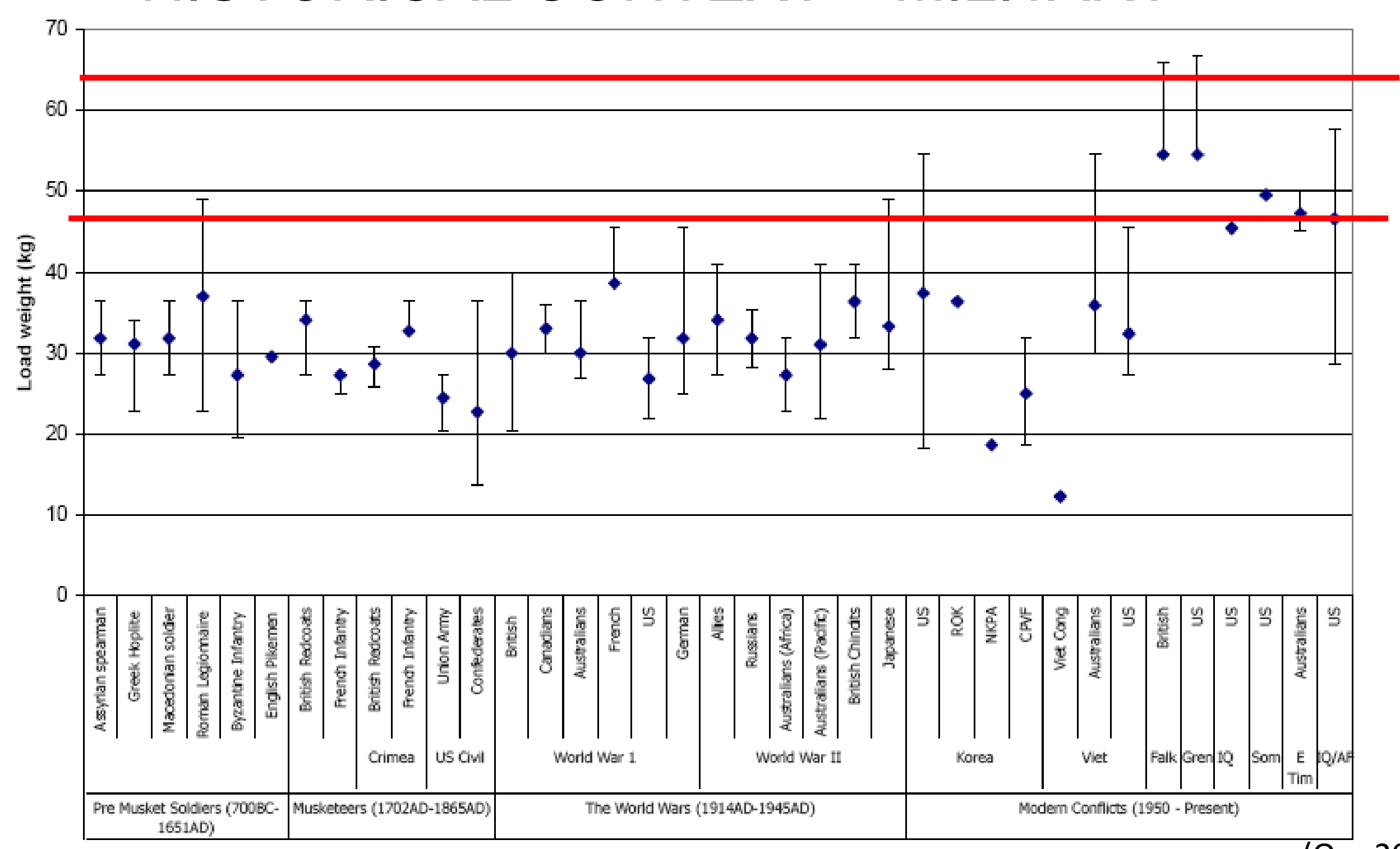




\section{CURRENT CONTEXT - AUSTRALIAN ARMY}

\section{On Operations (2001-2010)}

- PO loads

- $M=28.4 \pm 10.0 \mathrm{~kg}$

- heaviest mean load in $2008(\mathrm{M}=36.9 \pm 10.8 \mathrm{~kg})$

- MO loads

- $M=56.7 \pm 15.3 \mathrm{~kg}$

- heaviest mean load in 2009 ( $M=65.1 \pm 16.3 \mathrm{~kg})$

- OVERALL loads

- $47.7 \pm 21.0 \mathrm{~kg}$, (mean range over 10 years $=40.7 \mathrm{~kg}$ to $50.9 \mathrm{~kg}$ ), 


\section{CURRENT CONTEXT - AUSTRALIAN ARMY}

- Approximate relative load carried by Roman Legionnaires $=56 \%$

- Australian Soldiers in East Timor $=56 \%$

- US Soldiers in Afghanistan $=57 \%$
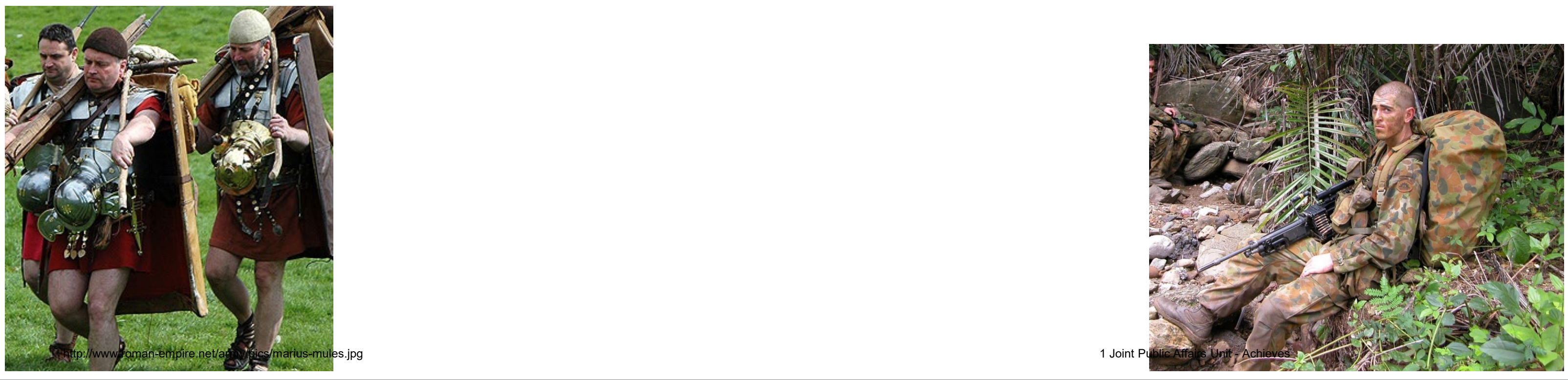


\section{ABSOLUTE VS RELATIVE LOADS}

- Currently female soldiers carry lighter absolute loads than male soldiers but only slightly heavier relative loads

\section{ABSOLUTE LOADS*}

FEMALE: $M=26.4 \mathrm{~kg}$

MALE: $M=39.0 \mathrm{~kg}$

$p=.045$ $\underline{\text { RELATIVE LOADS }}$

FEMALE: $M=43 \%$

MALE: $M=47 \%$

$p=.55$ 


\section{ABSOLUTE VS RELATIVE LOADS}

- Currently lighter soldiers carry the same absolute loads as heavier soldiers but heavier relative loads

\section{$\underline{A B S O L U T E L O A D S}$}

Light 20\%: $M=34.7 \mathrm{~kg}$

Heavy $20 \%: M=35.7 \mathrm{~kg}$

$\mathrm{p}=.902$
RELATIVE LOADS

Light $20 \%: M=49 \%$

Heavy $20 \%: M=36 \%$

$p=.0509$ 


\section{HISTORICAL CONTEXT - LEO}

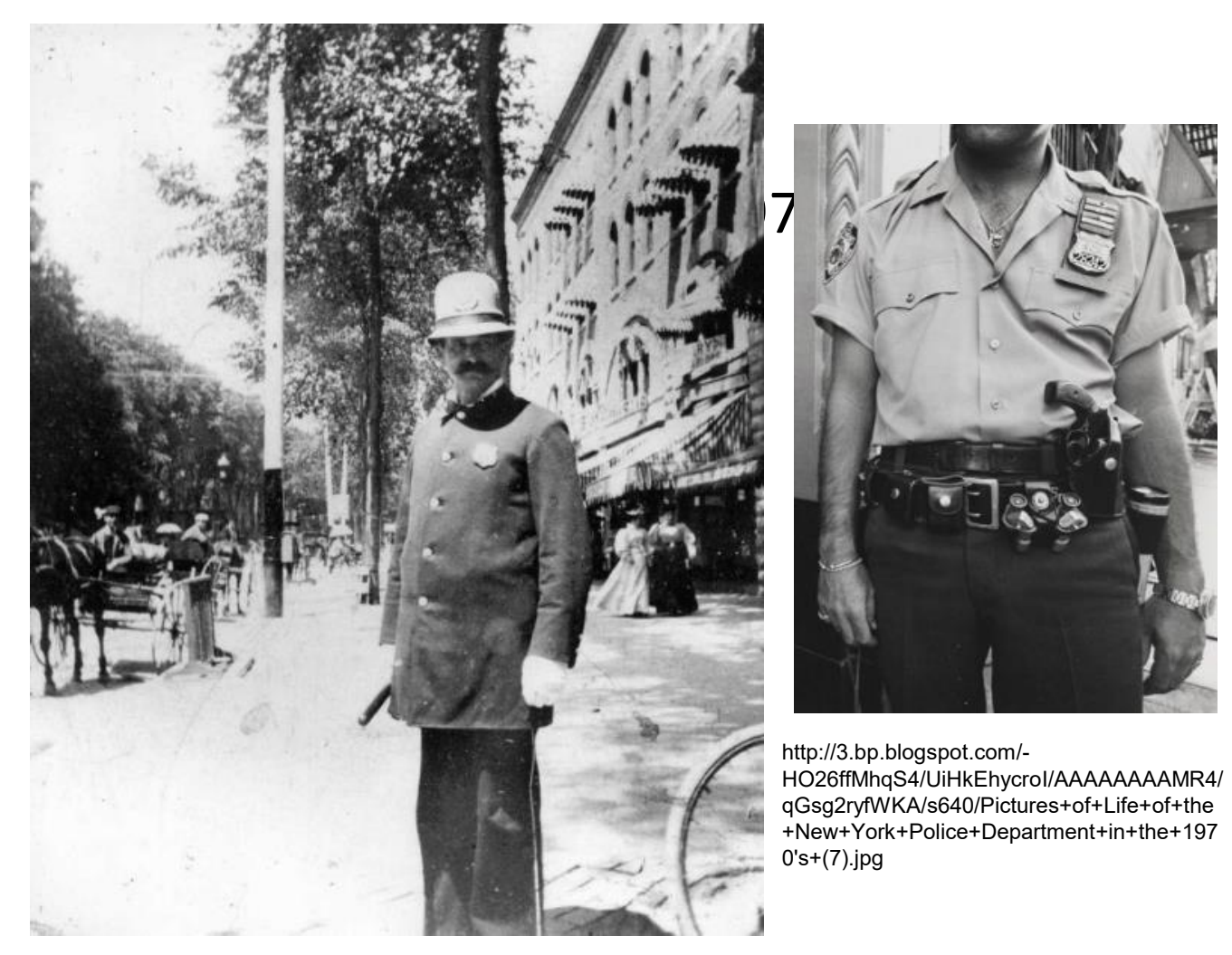

http://2.bp.blogspot.com/-

us+Black+\&+White+PhogAgraphs + of + The+Police+Officers +from +1890

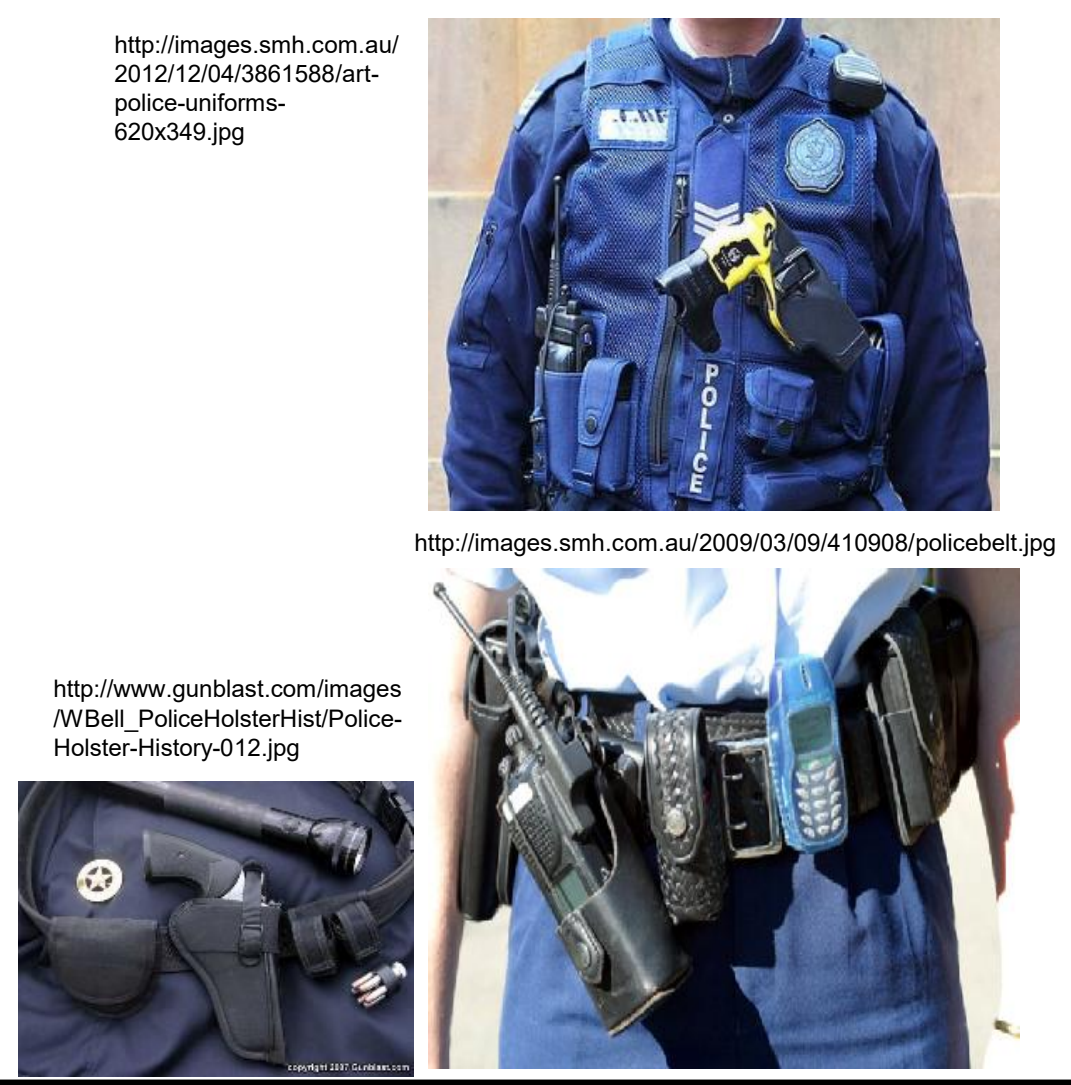

https://bond.edu.au/tru

列




\section{HISTORICAL CONTEXT - LEO}

- Police are becoming Christmas trees

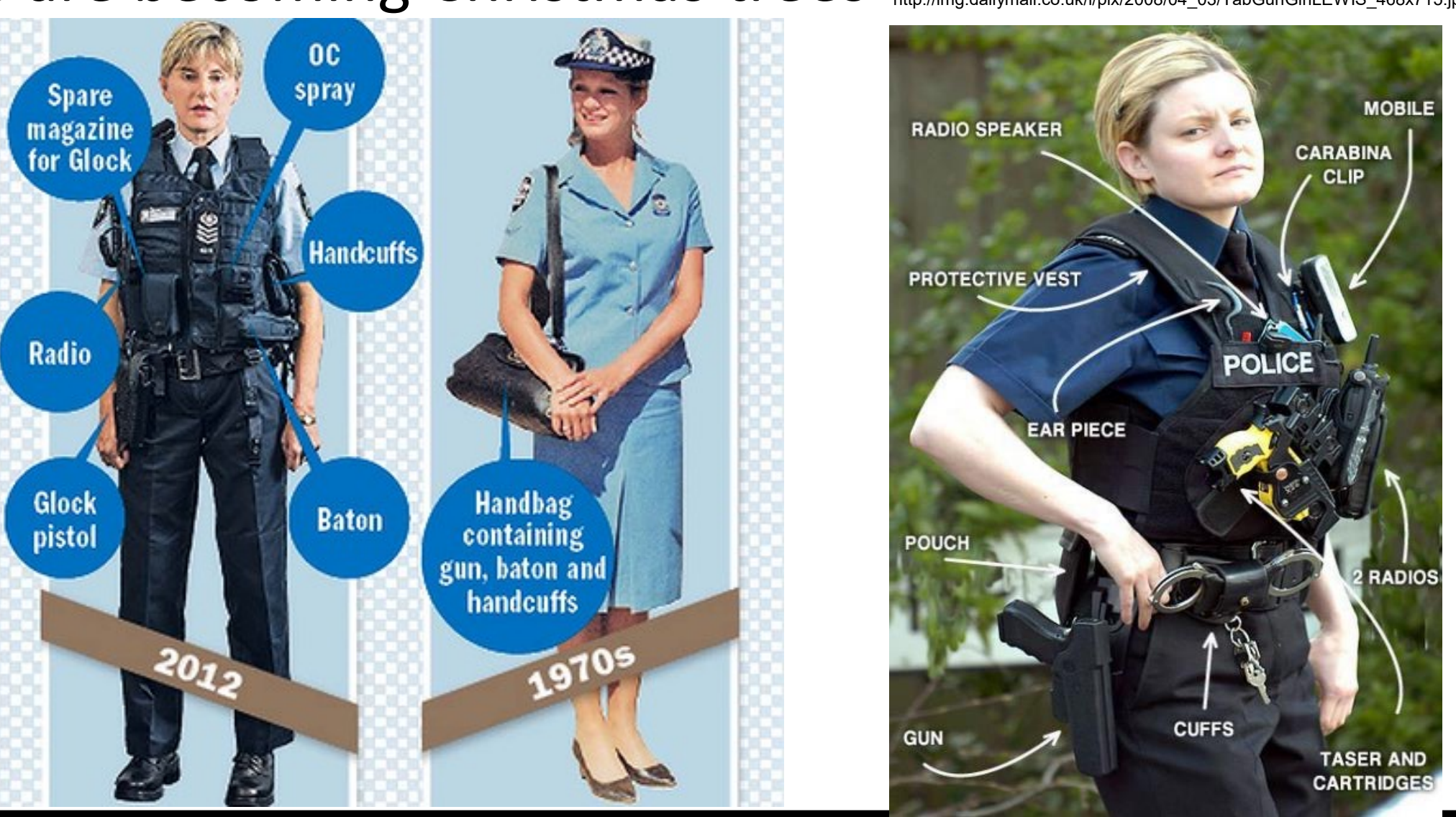




\section{BOND \\ UNIVERSITY \\ TACTICAL RESEARCH UNIT}

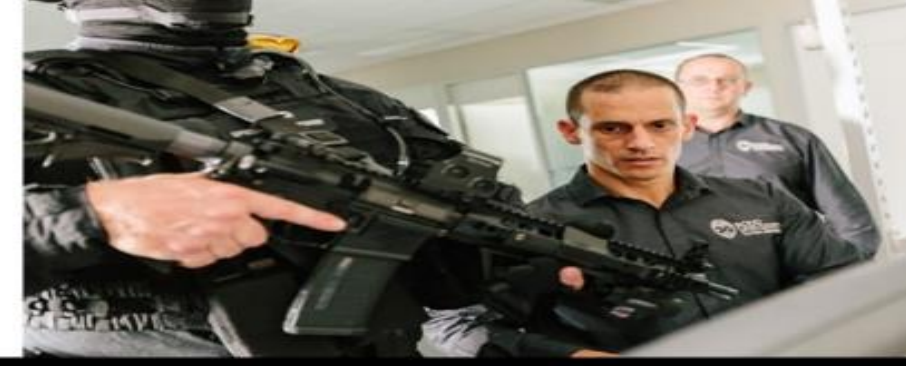

\section{HISTORICAL CONTEXT - LEO}

- Increasing levels of threat

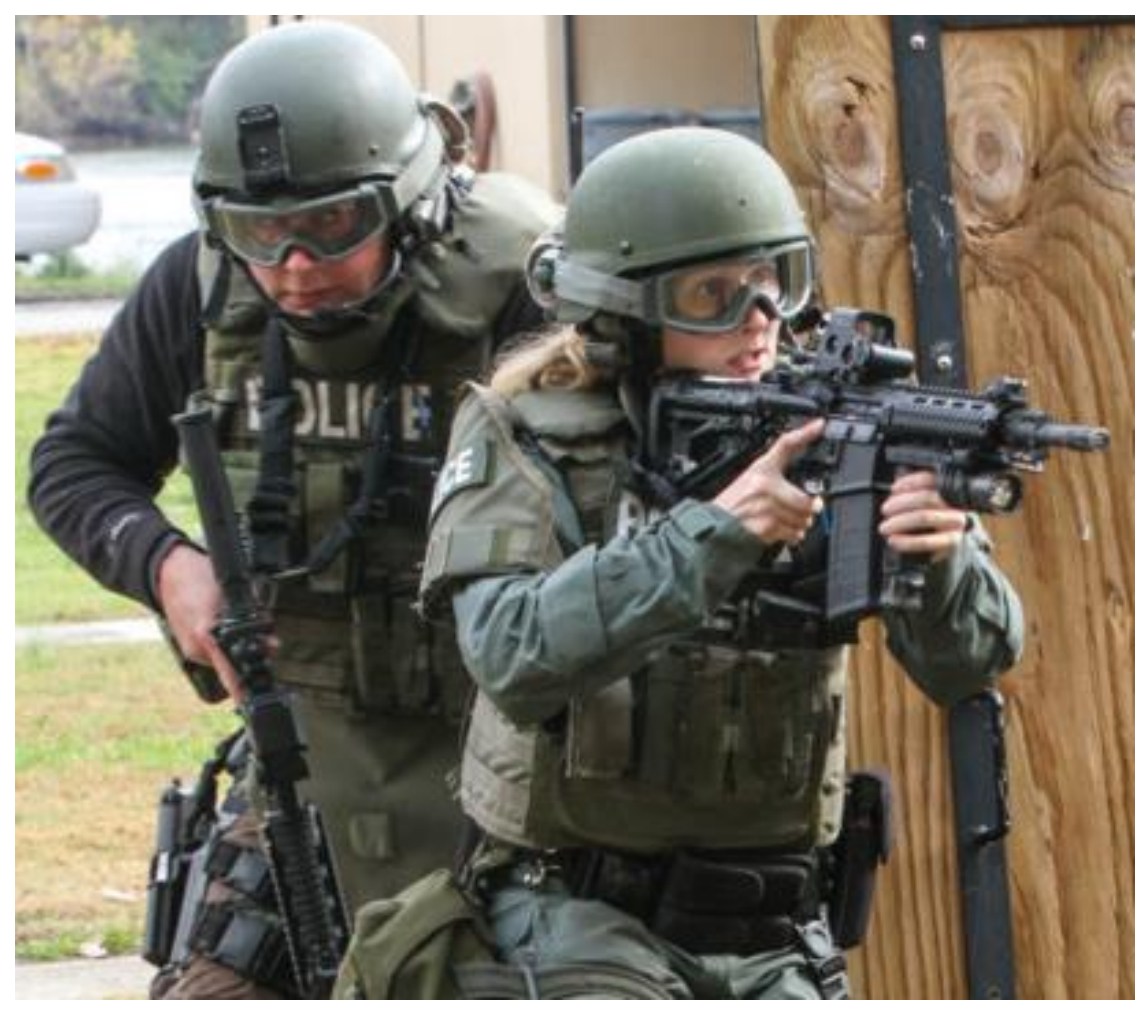




\section{HISTORICAL CONTEXT - AUSTRALIAN LEO}

\begin{tabular}{cccc}
\hline $\begin{array}{c}\text { ILAV type (A-C) \& } \\
\text { Normal station wear } \\
(\mathbf{N})\end{array}$ & $\begin{array}{c}\text { ILAV } \\
\text { Weight } \mathbf{( k g )}\end{array}$ & $\begin{array}{c}\text { Duty load } \\
\text { Complete } \\
\mathbf{( k g )}\end{array}$ & $\begin{array}{c}\text { Total load } \\
\text { including } \\
\text { officer weight } \\
\mathbf{( k g )}\end{array}$ \\
\hline A & $4.12 \pm 0.65^{*}$ & $11.53 \pm 0.77 \ddagger$ & $88.03 \pm 20.49$ \\
B & $3.54 \pm 0.70^{*}$ & $11.01 \pm 1.01 \ddagger$ & $87.51 \pm 20.60$ \\
C & $3.24 \pm 0.48^{*}$ & $10.77 \pm 1.16 \ddagger$ & $87.27 \pm 20.66$ \\
N & NA & $8.69 \pm 0.68$ & $85.19 \pm 20.24$ \\
\hline
\end{tabular}

* Significantly different $(p<0.05)$ between vests:

(Orr et al., 2016)

$\ddagger$ Significantly different $(\mathrm{p}<0.001)$ from normal station wear 


\section{CURRENT CONTEXT - AUSTRALIAN LEO}

\begin{tabular}{|c|c|c|c|c|}
\hline & FEMALE & MALE & FEMALE & MALE \\
\hline ILAV type & $\begin{array}{c}\text { ILAV + } \\
\text { Duty } \\
\text { Loads (kg) }\end{array}$ & $\begin{array}{c}\text { ILAV + Duty } \\
\text { Loads (kg) }\end{array}$ & $\% B W$ & $\% B W$ \\
\hline A & 11.14 & 11.85 & 16.90 & 14.90 \\
\hline B & 10.80 & 11.18 & 16.43 & 13.91 \\
\hline C & 10.24 & 11.22 & 15.60 & 13.95 \\
\hline $\mathrm{N}$ & 8.68 & 8.70 & 13.20 & 10.92 \\
\hline & \multicolumn{2}{|c|}{$*_{p}=0.225$} & \multicolumn{2}{|c|}{$*_{p}=0.009$} \\
\hline
\end{tabular}

(Orr et al., 2016) 


\section{ABSOLUTE VS RELATIVE LOADS}

- The LEO study found female officers carried the same absolute loads compared to the male officers

- However when expressed as a percentage of their body weight female officers carried significantly more relative load than male officers 


\section{CURRENT CONTEXT - AUTRALIAN LEO (TOU)}

Mean \pm SD Range

Absolute load carried $(\mathrm{kg})$

Relative load carried (\%BW)

(Carbone et al., 2014; Carlton et al., 2014)

\begin{tabular}{|c|}
\hline 1.8 \\
\hline $25.9 \pm 4.0$ \\
\hline
\end{tabular}
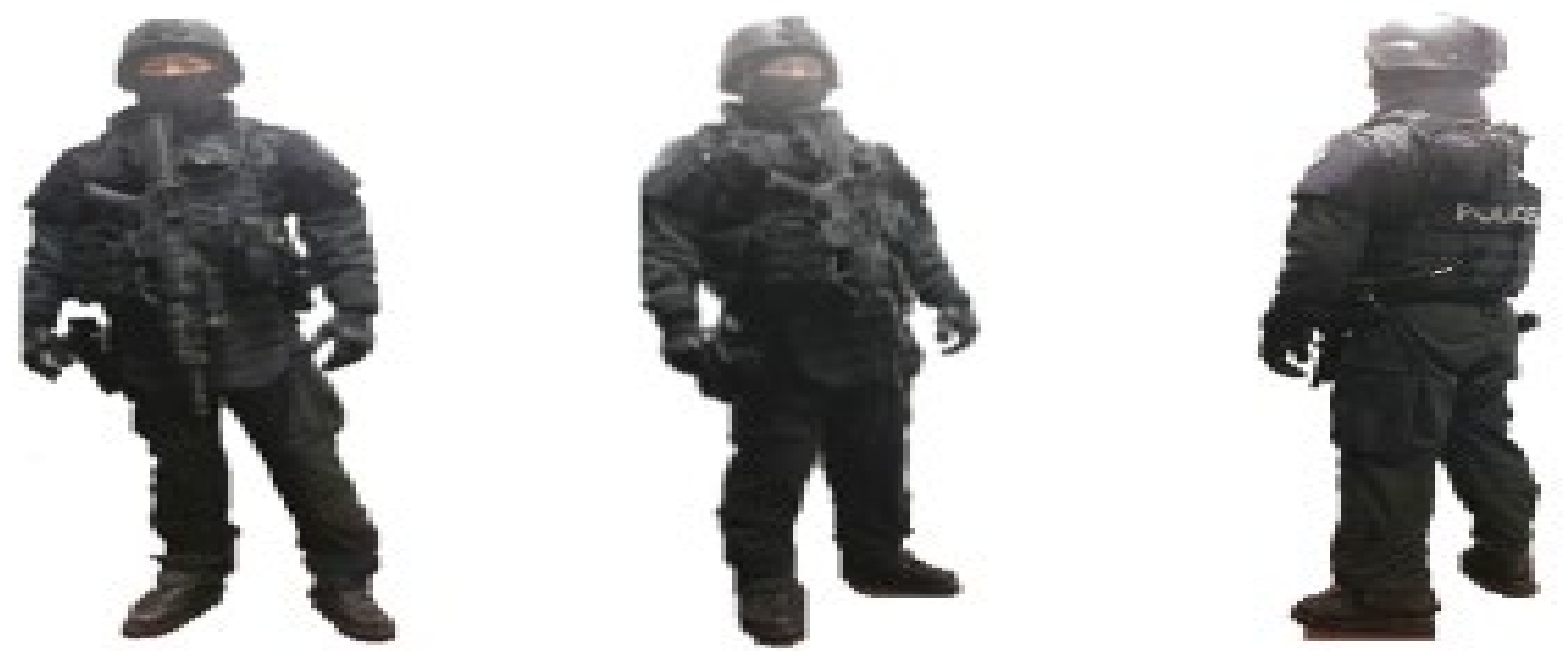


\section{SEX DIFFERENCES IN LC INJURIES}

Orr et al (2016).

- Mean ARA population over 2 years $=24,876$ personnel

- Female $n=2441$ (10\%): Male $n=22435$ (90\%)

- 401 reported injuries associated with load carriage

- Female $n=40$ (10\%): male $n=361$ (90\%)

- $R R=1.02(95 \% \mathrm{Cl} 0.74$ to 1.41$)$

- SPI

- Female $n=6(15 \%)$ : male $n=23(6 \%)$

- $\mathrm{RR}$ of $\mathrm{SPI}=2.40(95 \% \mathrm{Cl} 0.98$ to 5.88$)$

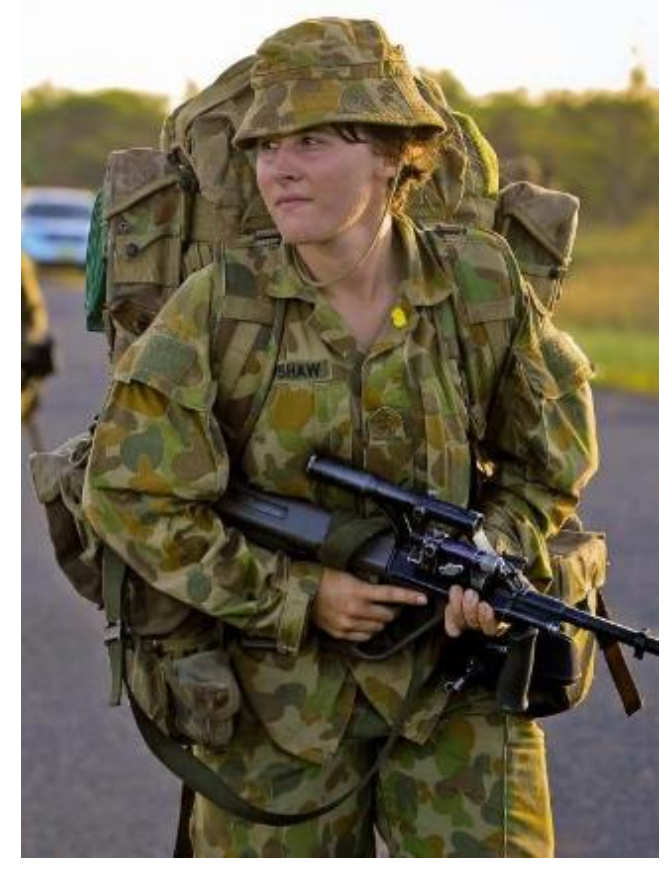




\section{IMPACTS ON PERFORMANCE - MARKSMANSHIP}

- Decrements in performance:

- $\downarrow$ Marksmanship (Knapik et al., 1990:1991:1997: Rice et al., 1999).

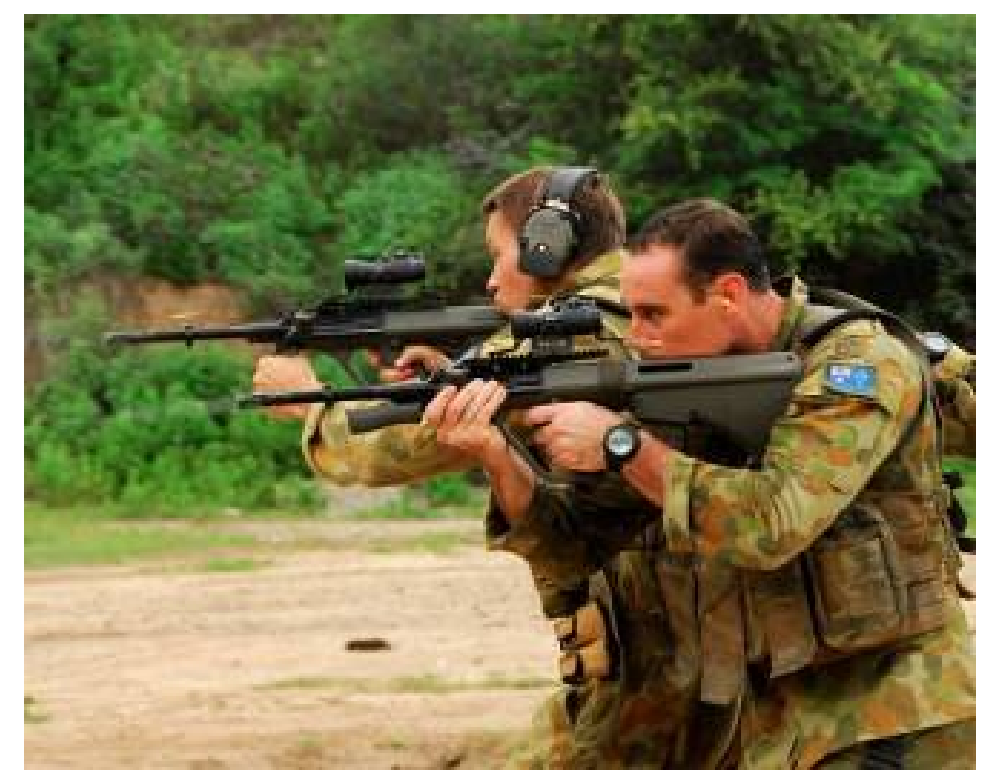




\section{IMPACTS ON PERFORMANCE - MARKSMANSHIP}

\section{- Reduced performance}

- Survey of 218 soldiers on operations

(Orr et al., 2013)

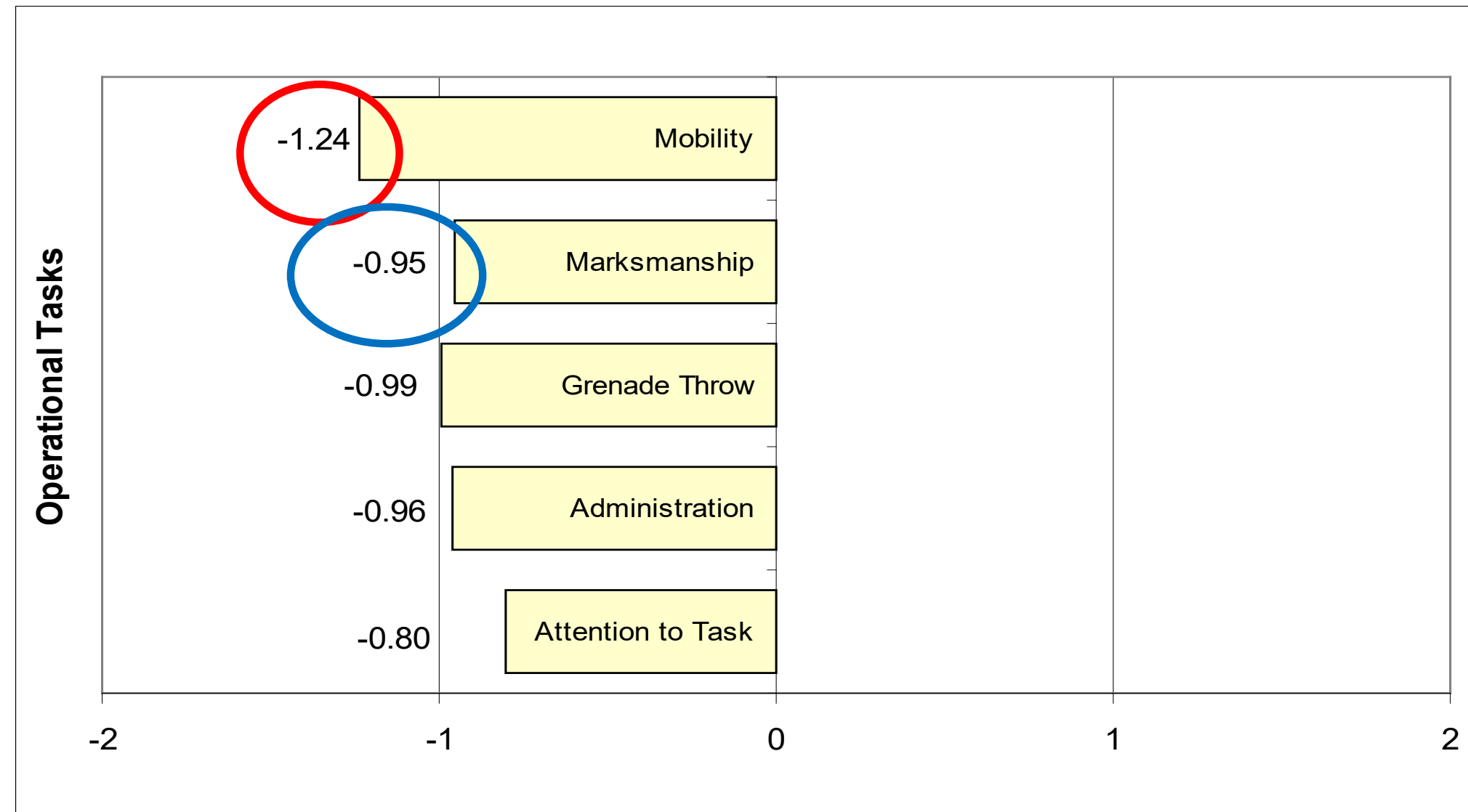




\section{IMPACTS ON PERFORMANCE - MARKSMANSHIP}

- Distance to centre of target

- DCOT

- Horizontal shot spread

- X-Dispersion

- Vertical shot spread

- Y-Dispersion

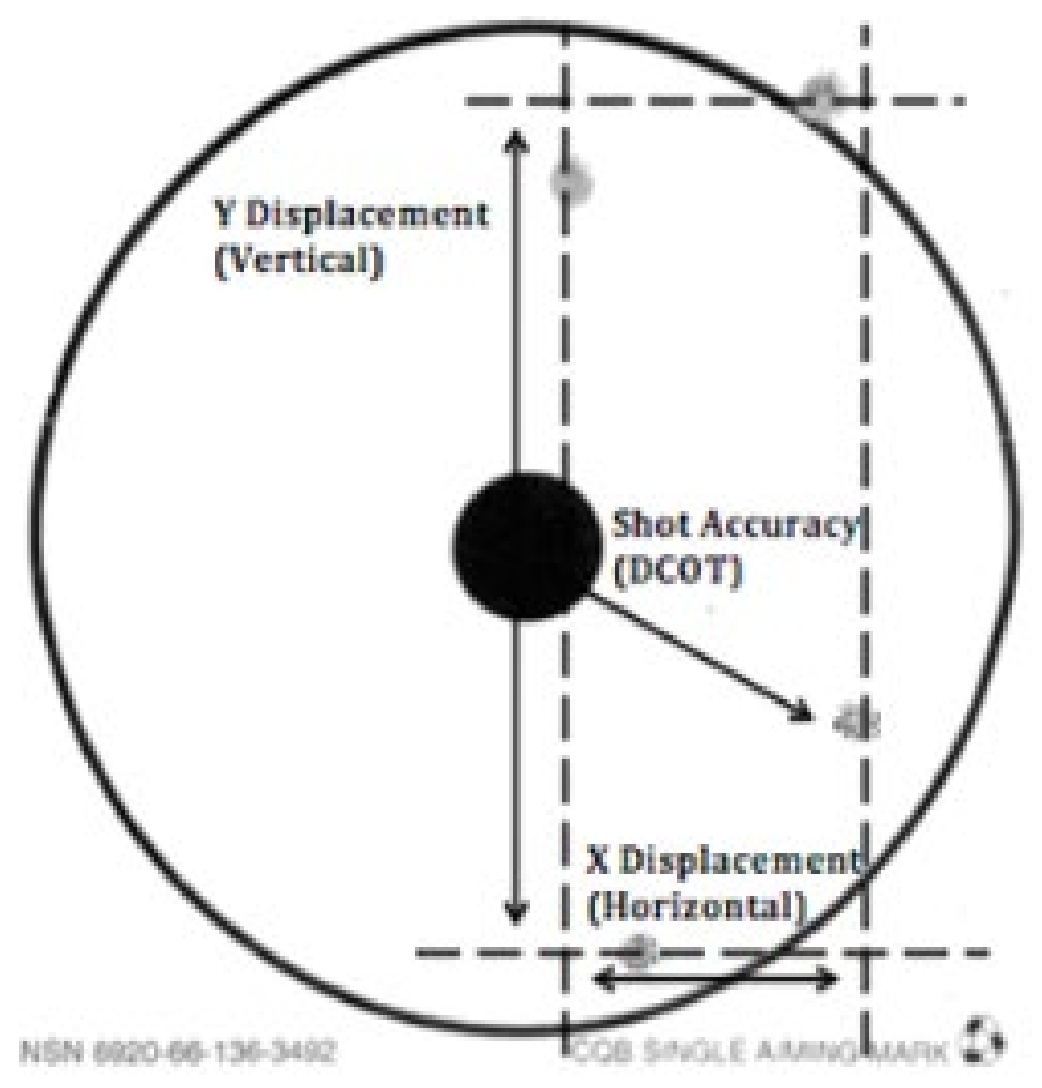

Carbone et al., 2014

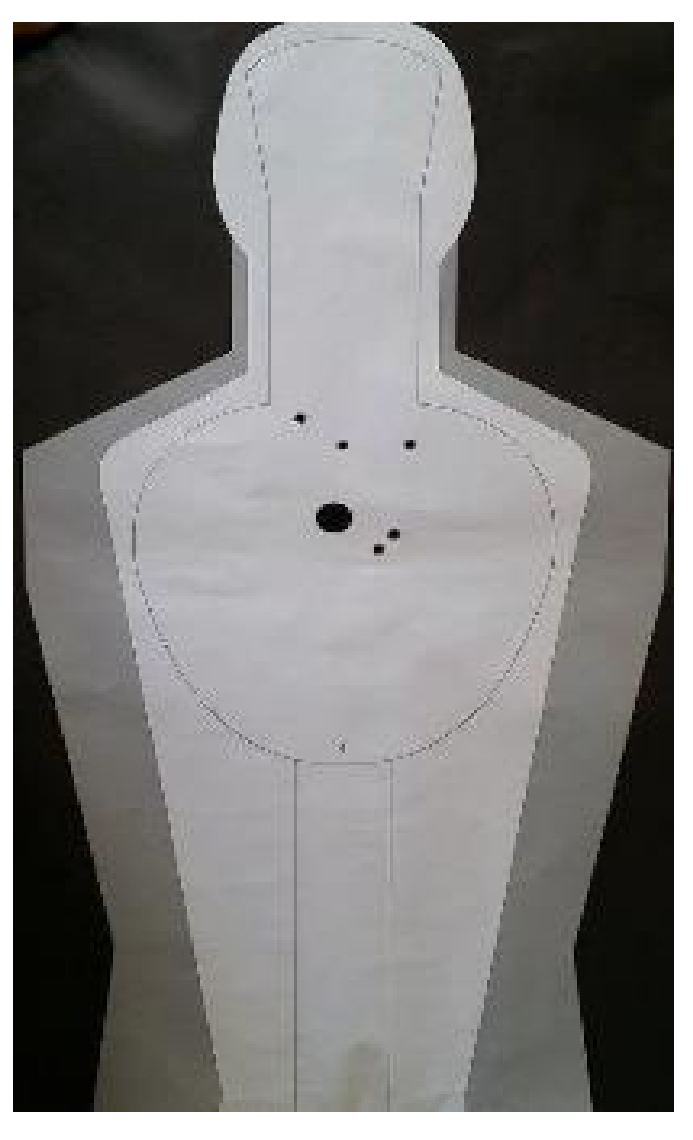




\section{IMPACTS ON PERFORMANCE - MARKSMANSHIP}

- Mobility Task

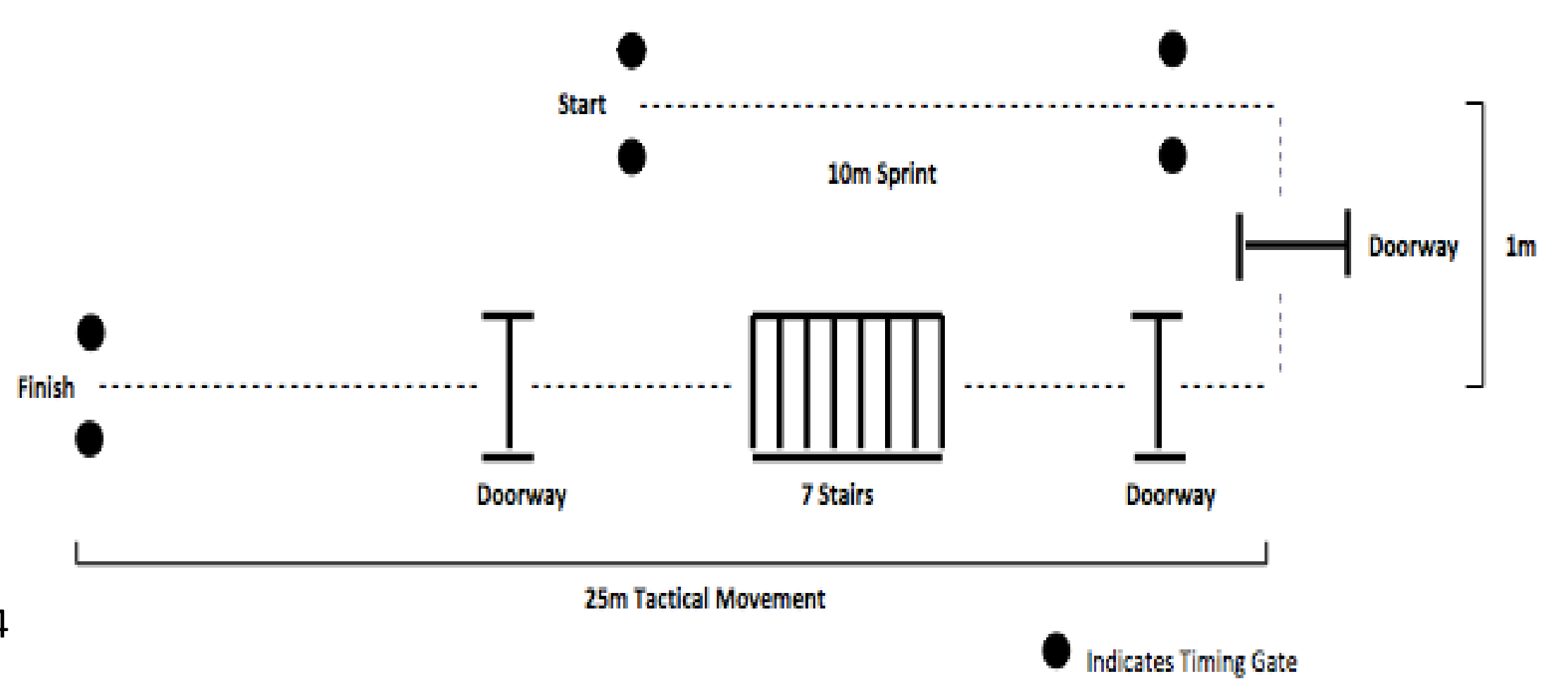




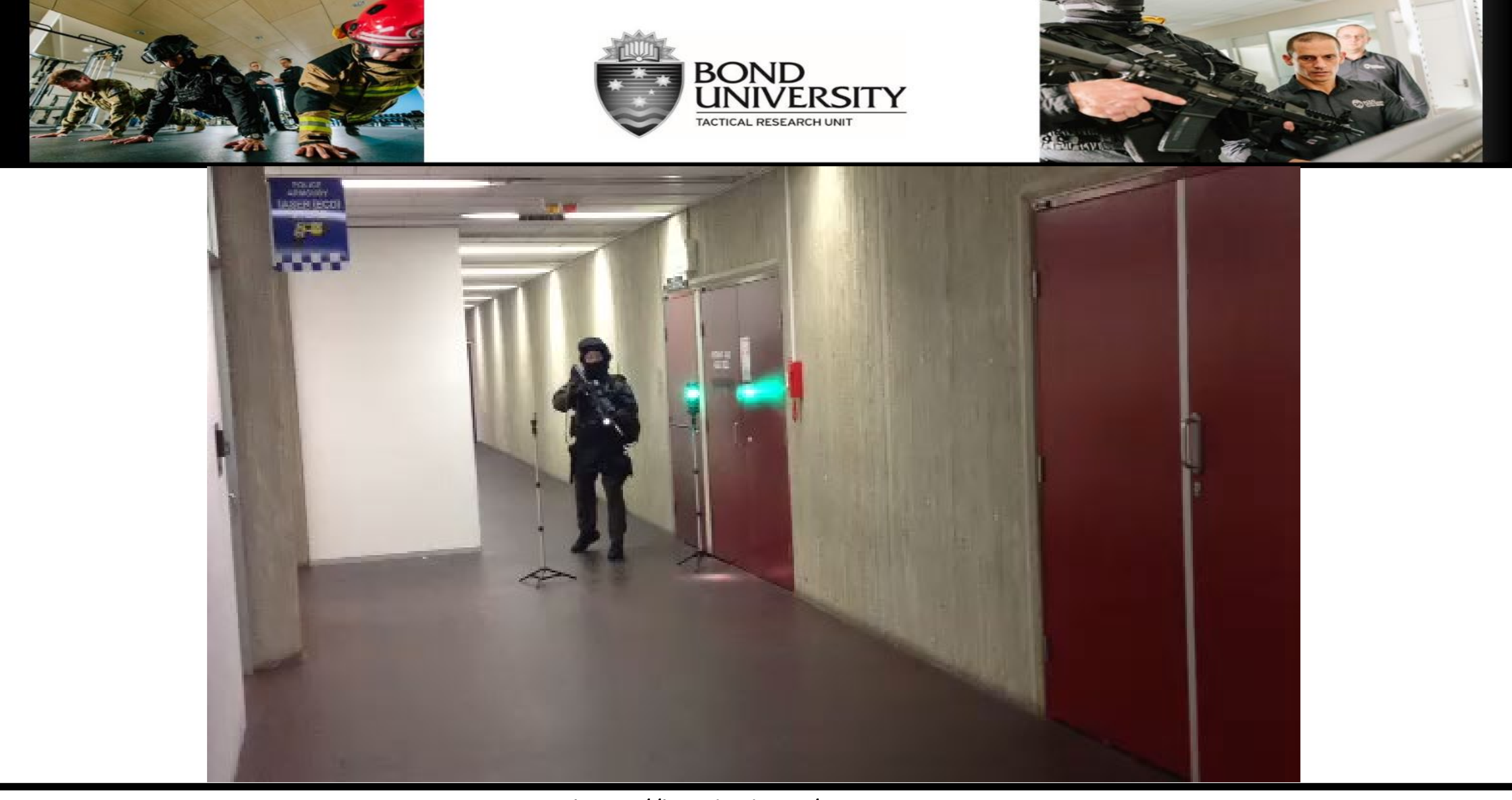




\section{IMPACTS ON PERFORMANCE - MARKSMANSHIP}

- Marksmanship

Carbone et al., 2014

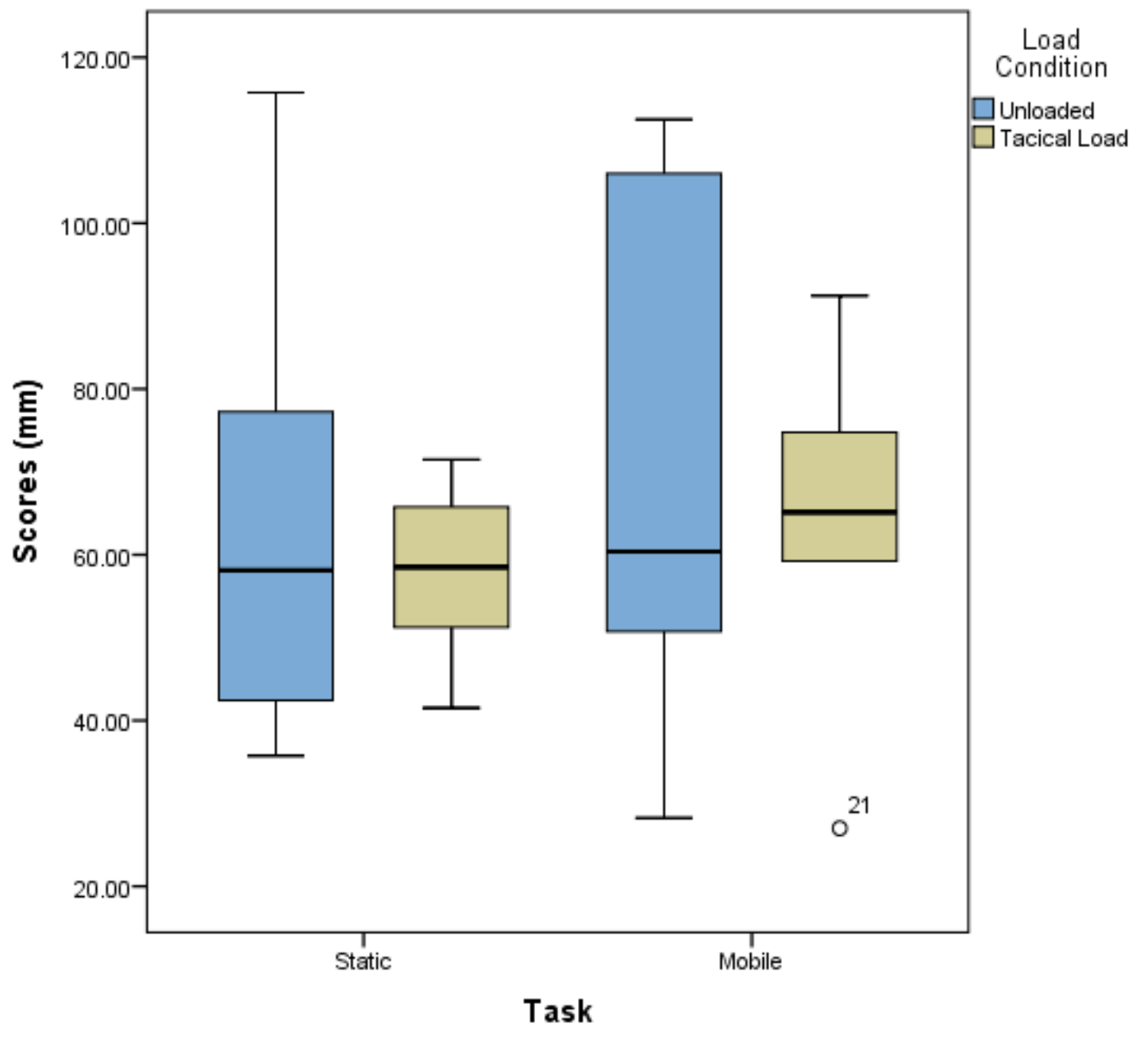



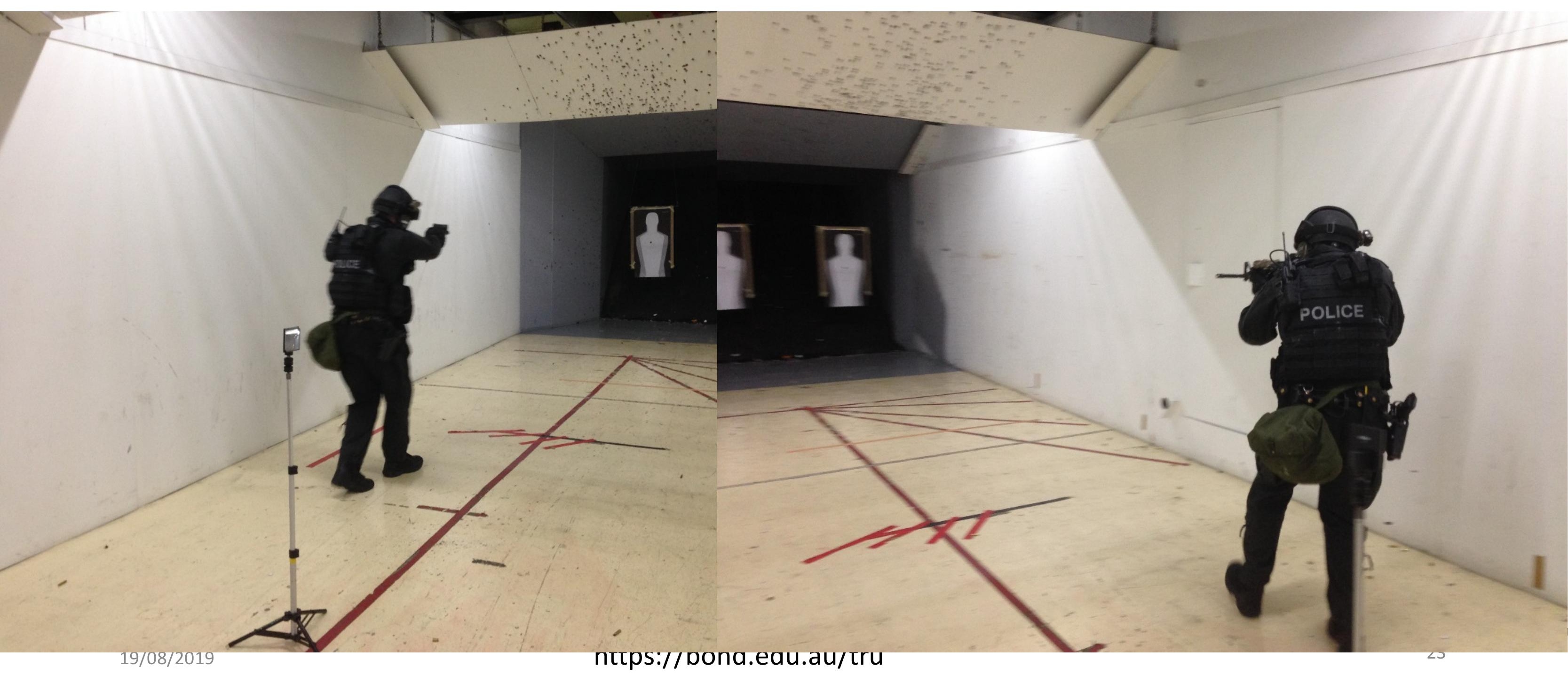


\section{IMPACTS ON PERFORMANCE - MARKSMANSHIP \\ Table 1. Primary weapon marksmanship results from all four conditions}

- No significant difference when TL

Orr et al., Unpublished

Task \& Loading Condition DCOT $(\mathrm{mm}) \quad$ X-Dlspersion $(\mathrm{mm}) \quad$ Y-Dispersion $(\mathrm{mm})$ Short Forward Movement

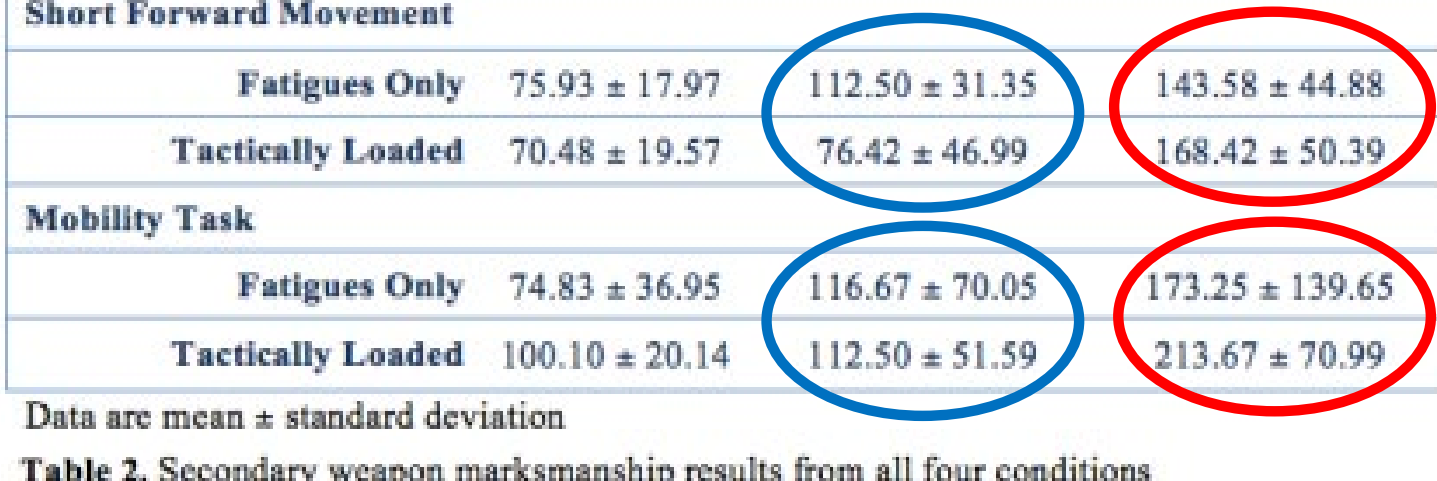

Table 2. Secondary weapon marksmanship results from all four conditions

\begin{tabular}{|c|c|c|}
\hline Task \& Loading Condition & DCOT $(\mathrm{mm})$ & X-Dispersilon (mm) Y-Dispersion (mm) \\
\hline \multicolumn{3}{|l|}{ Short Forward Movement } \\
\hline Fatigues Only & $107.35 \pm 37.68$ & $178.33 \pm 81$. \\
\hline Tactically Loaded & $112.60 \pm 44.37$ & \\
\hline \multicolumn{3}{|l|}{ Mobility Task } \\
\hline Fatigues Only & $128.23 \pm 33.20$ & $7.00=70$ \\
\hline Tactically Loaded & $108.70 \pm 52.48$ & $.08 \pm 13$ \\
\hline
\end{tabular}




\section{IMPACTS ON PERFORMANCE - MARKSMANSHIP}

\section{subpat Wumber 5}

\section{- Visual Analogue Scale (VAS)}

How do you think tactical load impacts on your marksmanship with the pistol when compared to carrying no load:
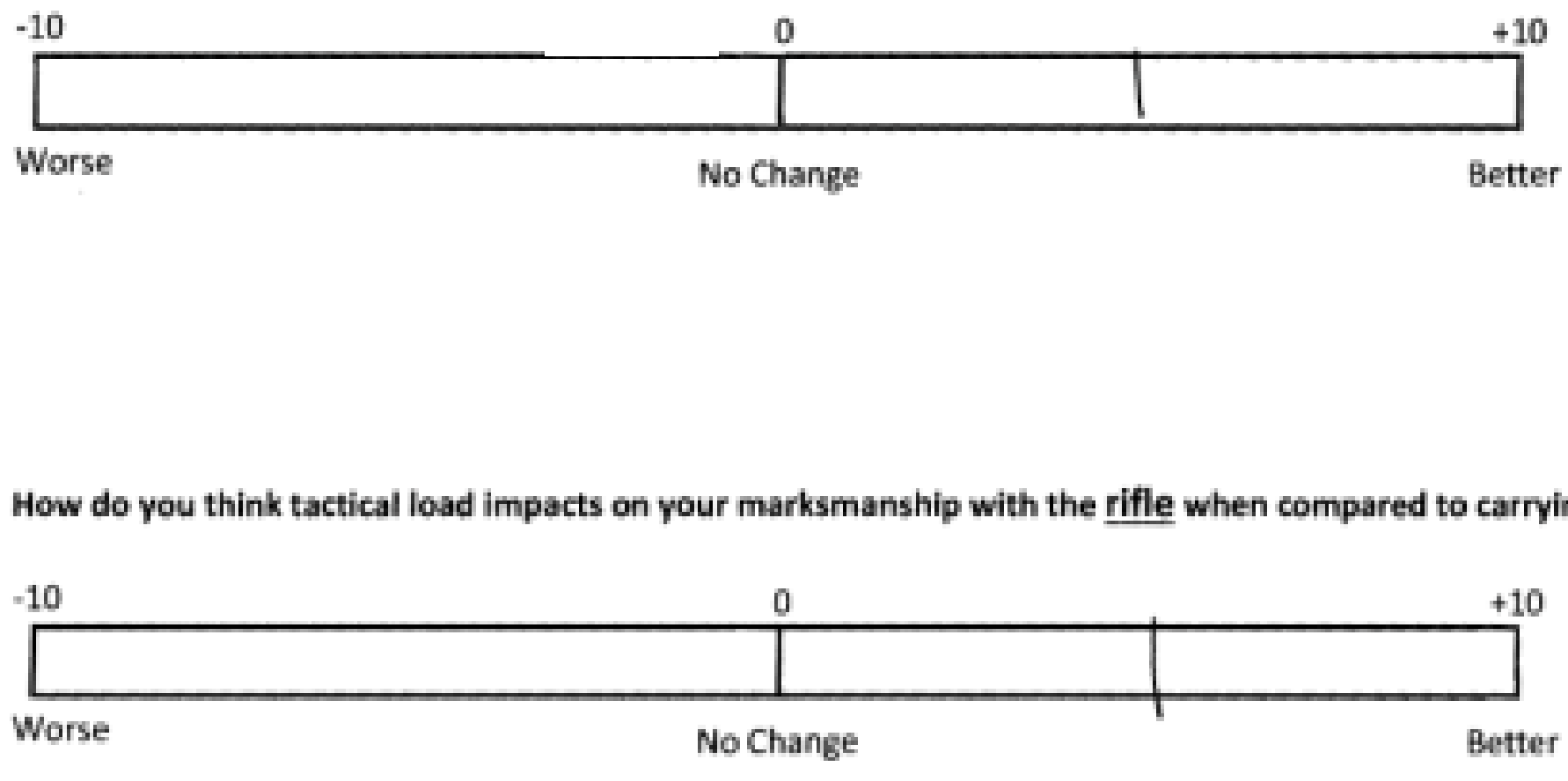


\section{IMPACTS ON PERFORMANCE - MARKSMANSHIP}

- Perceived significant improvement in marksmanship when TL

- Primary - VAS $+3.00 \pm 2.53(p=0.016)$

- Secondary - VAS $+2.83 \pm 2.93,(p=0.039)$

Correlations between perceptions of load carriage impacts on performance and actual marksmanship scores

- Primary: Short move: $r=-0.347,(p=0.500)$ and mobility task: $r=-0.401(p=0.431)$

- Secondary: Short move: $r=-0.631(p=0.179)$ and mobility task: $r=-0.306,(p=0.555)$ 


\section{IMPACTS ON PERFORMANCE - MARKSMANSHIP}

- GD police $(n=11)$

- Average marksmanship scores $(p=.118)$

- ILAV B - smallest SD,

- $\quad$ ILAV A: a negative impact, $-2.1(95 \% \mathrm{Cl}-5.5$ to +1.3)

- $\quad$ ILAV B: a positive impact, $+2.7(95 \% \mathrm{Cl}+0.4$ to +5.0$)$

- $\quad$ ILAV C: a negative impact, $-1.7(95 \% \mathrm{Cl}-4.4$ to +0.9$)$

- Normal station wear: a positive impact, $+1.4(95 \% \mathrm{Cl}-2.2$ to +5.0$)$ 


\section{IMPACTS ON PERFORMANCE - MOBILITY}

- Decrements in performance:

- $\downarrow$ Mobility

- Impeded mission success (Breen 2000)
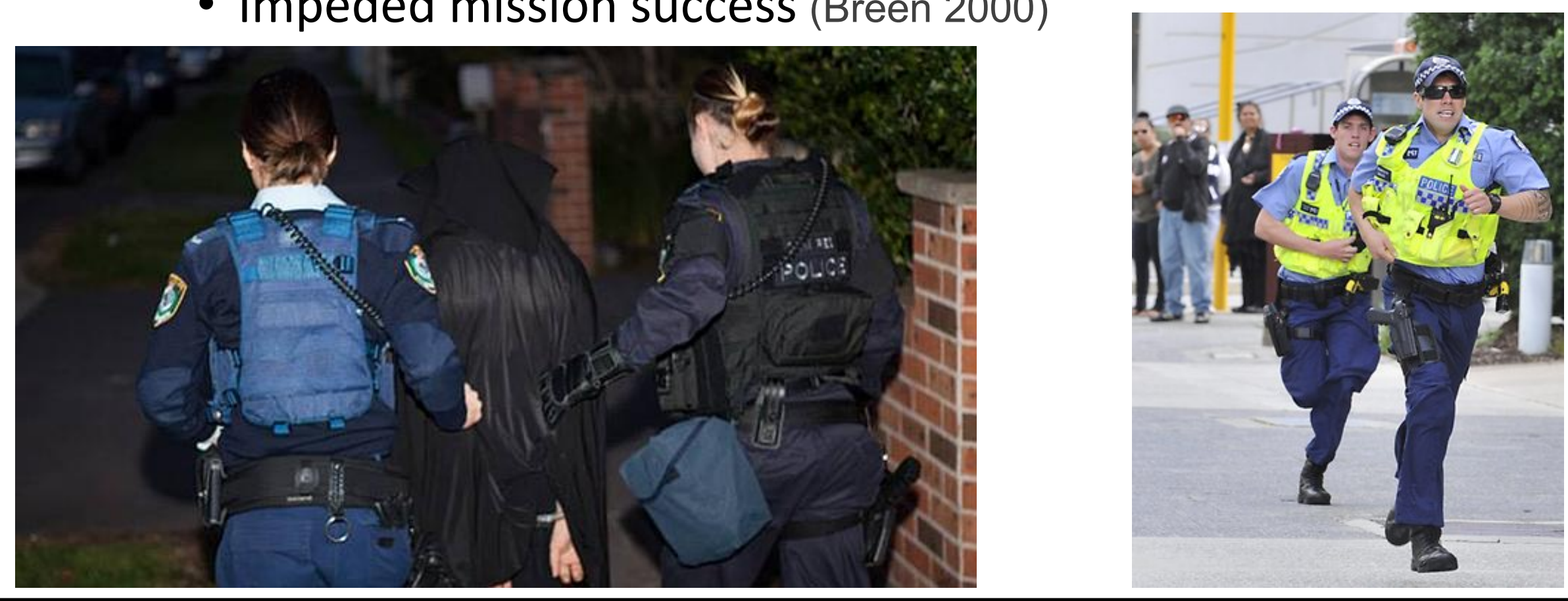
IMPACTS ON PERFORMANCE - MOBILITY

- Victim Drag (10m)

- Police Vehicle Exit and Sprint

Victim Drag

Condition

ILAV A

ILAV B

ILAV C

$\mathrm{N}$
Time (s)

$5.74 \pm 0.28$

$5.47 \pm 0.23$

$5.50 \pm 0.38$

$5.56 \pm 0.43$
Vehicle Exit

Time (s)

$3.49 \pm 0.94$

$3.41 \pm 0.87$

$3.40 \pm 1.06$

$3.41 \pm 0.85$ 


\section{IMPACTS ON PERFORMANCE - MOBILITY}

$\begin{array}{lll} & \text { Unloaded } & \text { Loaded } \\ \text { 10m sprint (sec) } & 2.40 \pm 0.22 & 2.46 \pm 0.15 \\ \text { 10m dummy drag (sec) } & 6.89 \pm 0.44 & 7.79 \pm 0.75^{*} \\ \text { Total time (sec) } & 9.29 \pm 0.53 & 10.25 \pm 0.77^{*}\end{array}$

* Indicates statically significant differences between unloaded and loaded, $p<0.01$.

Carlton et al., 2014 


\section{ENCAPSULATION}

- Loads for both LEO and Army are increasing

- Female soldiers carry lighter absolute but similar relative loads

- Female LEO carry similar absolute but heavier relative loads

- There are differences in injuries sustained based on sex

- There are different impacts of load on marksmanship (primary / secondary weapon)

- Soldiers think load reduces marksmanship, LEO varies but appear accurate

- Load impacts on mobility - but the load may need to reach a threshold 


\section{BOND \\ UNIVERSITY \\ TACTICAL RESEARCH UNIT}

\section{Australian Tactical Loads and their Operational Impacts}

\section{References avail on request from tru@bond.edu.au}

$$
\text { Science and Technology }
$$

$4^{\text {th }}$ International Congress on Soldiers' Physical Performance 28 November - 1 December 2017

Melbourne Australia

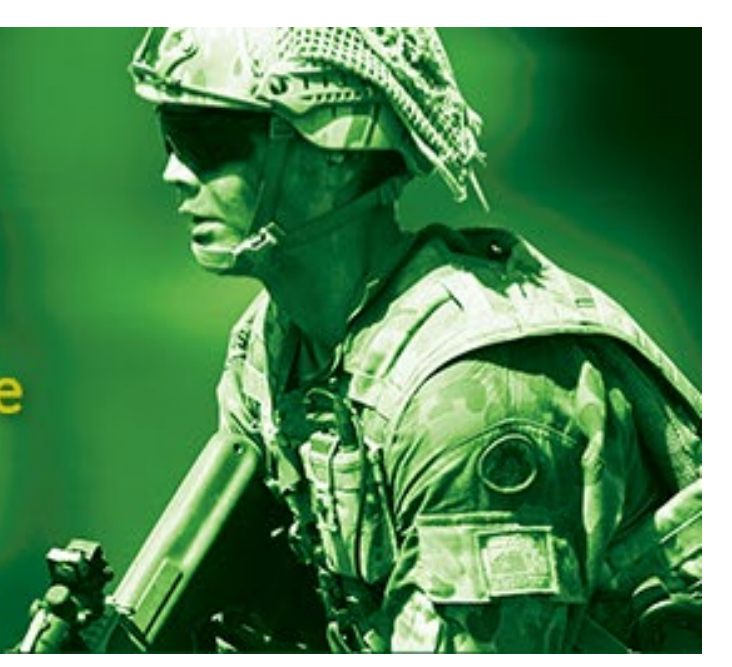

